\begin{tabular}{|c|l|}
\hline Title & The effect of exogenous spatial attention on auditory information processing. \\
\hline Author(s) & Kanai, Kenichi; Ikeda, Kazuo; Tayama, Tadayuki \\
\hline Citation & $\begin{array}{l}\text { Psychological Research, 71(4), 418-426 } \\
\text { https:/doi.org/40.1007/300426-005-0024 4 }\end{array}$ \\
\hline Issue Date & 2007-07 \\
\hline Doc URL & http://hdl.handle.net/2115/26441 \\
\hline Rights & The original publication is available at www.springerlink.com \\
\hline Type & article (author version) \\
\hline File Information & PRPF71-4.pdf \\
\hline
\end{tabular}

Instructions for use 


\section{The effect of exogenous spatial attention on auditory information processing}

Kenichi Kanai

Information Security Unit, NTT Advanced Technology Corporation, 12--1, Ekimae-Honcho, Kawasaki, Kanagawa, 210-0007,

Kazuo Ikeda

Department of Human Culture, Kochi University, 2-5-1 Akebonocho, Kochi, 780-8520,

Tadayuki Tayama

Department of Psychology, Hokkaido University, N10 W7, Kita-Ku, Sapporo, Hokkaido, 060-0810,

Correspondence:

Tadayuki Tayama

Department of Psychology, Hokkaido University, N10 W7, Kita-Ku, Sapporo, 060-0810, Japan

Tel: +81-011-706-4199

E-mail: tayama@psych.let.hokudai.ac.jp 


\section{Abstract}

This study investigated the effect of exogenous spatial attention on auditory information processing. In Experiments 1, 2 and 3 , temporal order judgment tasks were performed to examine the effect. In Experiment 1 and 2, a cue tone was presented to either the left or right ear, followed by sequential presentation of two target tones. The subjects judged the order of presentation of the target tones. The results showed that subjects heard both tones simultaneously when the target tone, which was presented on the same side as the cue tone, was presented after the target tone on the opposite side. This indicates that spatial exogenous attention was aroused by the cue tone, and facilitated subsequent auditory information processing. Experiment 3 examined whether both cue position and frequency influence the resulting information processing. The same effect of spatial attention was observed, but the effect of attention to a certain frequency was only partially observed. In Experiment 4, a tone fusion judgment task was performed to examine whether the effect of spatial attention occurred in the initial stages of hearing. The result suggests that the effect occurred in the later stages of hearing.

\section{Introduction}

Our experiences suggest that we can intentionally attend to a sound from a certain direction. Such auditory attention is directed automatically to the location of a suddenly emitted sound. This kind of auditory attention toward a certain direction is called spatial attention. In studies of vision, focal attention in a space has been considered to be essential for feature integration, as described in the feature integration theory (Treisman \& Gerade, 1980), while spatial attention in audition has not necessarily 
been regarded as an indispensable factor. This may be because visual information is structured retinotopically, whereas the auditory information is structured tonotopically (Buchtel \& Butter, 1988). However, spatial attention may still play a role in audition. Indeed, some previous studies have suggested that spatial attention does influence auditory information processing (see Scharf, 1998).

The findings of preceding studies that examined auditory spatial attention have been controversial. Several studies have reported that spatial attention influences reaction times and sensitivities in spatial discrimination tasks (Rhodes, 1987; Spence \& Driver, 1994; Quinlan \& Bailey, 1995; McDonald \& Ward, 1999; Sach, Hill \& Baily, 2000), whereas it does not affect reaction times in detection tasks (Posner, 1978; Scharf, Quigley, Aoki, Peachey \& Reeves, 1987; Buchtel \& Butter, 1988; Spence \& Driver, 1994). Rhodes (1987) argued that the simple detection tasks by studies of audition could be performed through the non-spatial representation at early stages of the auditory system, thereby resulting in performance being unaffected by auditory spatial attention. By conducting a spatial discrimination experiment performed at a higher level of the auditory system, Rhodes (1987) found that spatial attention influenced the reaction time. However, the discrepancy between these results might be caused by other methodological problems in the experiments. Bregman (1990) pointed out that reaction times in the spatial discrimination tasks might reflect interactive effects between response biases and attention, because the conditions of Rhodes's experiment (1987) could affect not only the perceptual processing but also the motor processing of responses. The same interpretation can be applied to the experiments executed by Quinlan and Bailey (1995). Spence and Driver (1994) used the orthogonal cuing paradigm to avoid the response biases and showed the effect of auditory spatial attention. Nevertheless, McDonald 
and Ward (1999) claimed that listeners in spence and Driver's experiment (1994) might have judged the elevation of the target relative to that of the cue. They conducted the experiments using the go-no-go task, in which listeners were instructed to respond to peripheral (left or right) targets (go trials) and not to respond to the central target (no-go trials). This revealed the effect of spatial exogenous attention on this task.

Though most of these previous studies have used reaction time to examine the effect of auditory spatial attention, the aforementioned points highlight that it is not necessarily a suitable measure. To investigate the effects of auditory attention, we focused on another experimental paradigm; the temporal order judgment (TOJ) task paradigm. This paradigm has been often used in cross-modal studies. The typical result is that attending to a sensory modality speeds up the perception of stimuli in that modality (Shore, spence, \& Klein, 2001). This effect has been known as the prior entry effect (Titchener, 1908). This paradigm was also used to examine the effects of spatial attention in studies of vision (Stelmach \& Herdman, 1991; Jaskowski, 1993; Gibson \& Egeth, 1994). Stelmach \& Herdman (1991) asked participants to judge the temporal order of two visual target stimuli just after a cue stimulus. Results revealed that a target stimulus coming from the same direction as the cue stimulus appeared before the other target stimulus from a different direction. They suggested that the exogenous attention was aroused by the cue stimulus, and the directed attention affected the information transmitting of the target stimulus.

Since the TOJ task does not require a rapid response, it has been said that the TOJ task can reveal the subtle effects of attention on the speed of information processing (Sternberg \& Knoll, 1973) without the influence of motor factors. However, recent studies have suggested that the prior entry effects found by the TOJ tasks have been confounded by response bias (Pashler, 
1998; Shore, Spence, \& Klein, 2001 ; Spence, Shore, \& Klein, 2001$)$. Spence, Shore, \& Klein (2001) insisted that all previous studies demonstrating multisensory prior entry may reflect nothing more than response bias or may reflect some unknown combination of response bias and attentional effects. Shore, Spence, \& Klein (2001) and Spence, Shore, \& Klein (2001) introduced a novel methodology to minimize the effect of response bias by manipulating attention and response demands in orthogonal dimensions and showed the existence of multisensory prior entry by using this methodology.

Thus, the effect of spatial exogenous attention on the TOJ tasks might also be contaminated by response bias. However, we consider that the TOJ task has an advantage over the reaction time task in circumventing the problem of response biases, from the point that it does not require a rapid response. If we could reduce the influences of response biases and carefully inspect the results, this task would be suitable to examine this effect. We do not have enough data to discuss the effects of auditory spatial attention on the TOJ task. Therefore, we will adopt the TOJ task in the present study and investigate the effects of exogenous attention on the speed of auditory information processing by this task.

Previous studies showed that auditory attention oriented to a certain direction facilitated the processing of a stimulus presented from the same direction (Rhodes, 1987; Spence \& Driver, 1994; Quinlan \& Bailey, 1995; McDonald \& Ward, 1999). According to these findings, listeners will perceive the target tone on the same side as the cue tone before the target tone on the opposite side, even if both target stimuli are presented simultaneously. In Experiment 1 and 2, we examined whether auditory attention facilitated auditory information processing by implementing the TOJ task. Participants judged which target was presented first (Experiment 1) or which target was presented second (Experiment 
2). In Experiment 3, we compared the attention effects of spatial consistency with those of frequency consistency by using the same task. In these experiments, we examined the effective duration of exogenous attention by manipulating the cue leading time. In Experiment 4, a tone fusion judgment task was conducted to examine in which stage of the auditory system the effects of exogenous spatial attention occur.

\section{Experiment 1}

Method

Participants

Five undergraduates and postgraduates participated in the experiment. All participants had normal hearing ability.

Apparatus and materials

All stimuli were generated digitally to 16 -bit resolution at a sampling of $22.5 \mathrm{KHz}$ by using the $\mathrm{D} / \mathrm{A}$ converter in a personal computer (Apple, Power Macintosh 7600/200). All cue and target tones were pure tones of $1000 \mathrm{~Hz}$ and $20 \mathrm{msec}$ which were presented at $86 \mathrm{~dB}$ SPL through a headphone (Trio, KH-71). All responses were recorded by personal computer through its keyboard.

Experimental condition

The experiment was performed under two task conditions: no-cue condition and cue condition. In both conditions, the TOJ tasks were performed. Two target tones were presented to left and right ears with eight kinds of stimulus onset asynchrony (SOA) : -60, 
$-40,-20,-10,10,20,40$ and $60 \mathrm{msec}$. The positive SOA was arbitrarily assigned to a situation where the right target was presented before the left target. The negative SOA indicates the reverse. In the cue condition, a cue tone was additionally presented just before the target tones (see Fig. 1). There were two kinds of variables with cue tones: cue position (CP; left and right) and cue leading time (CLT). The CLT is the variable of asynchrony between the onsets of the cue tone and the first target tone. There were four levels of CLT: 150, 200, 400, and $800 \mathrm{msec}$. As a repeated measures design was adopted, participants received 64 trial conditions, composed of SOA (8 levels), CP (2 levels) and CLT (4 levels) in the cue condition.

Procedure

Participants were tested individually. Each participant wore a headphone and a blindfold and was seated in a sound-attenuated chamber. They initiated each trial by pressing a space key. After $800 \mathrm{msec}$, two targets were presented. Under the cue condition, cue tone was presented before the two targets. They were instructed to ignore the cue tone and judged the temporal order of the two targets by pushing one of two keys. They press the right key if they heard the right target first, and press the left key if they heard the left target at first. The experiment was composed of seven sessions. The first session was a practice session for the cue condition, where 80 trials were performed with feedback of accuracy. The feedback was not given in the subsequent experimental sessions. The next two sessions were experimental sessions of the no-cue condition, where 80 trials (10 trials $x$ 8 SOAs) were performed for each session. The next four sessions were experimental sessions of the cue condition, where 320 trials ( 5 trials $x 8$ SOAs $x 2$ CPs x 4 CLTs) were performed for each session. The presentation order of experimental conditions was randomized 
in each session. Throughout all experimental sessions, each participant performed 20 trials under the same condition.

Results and Discussion

The percentage of the right (first) responses was calculated for each condition across all participants. The values were transformed into $z$-scores and were plotted as a function of SOA. The function of the regression line was determined by the method of least squares, and the point of subjective synchrony (PSS) was estimated by calculating the value of SOA at the point where the $z$-score equals zero on the regression line. The mean PSS across participants for the no-cue condition was -1.1 msec. This indicates that participants have high abilities for the performance on this task. The mean PSSs for right CP for the cue condition were multiplied by -1.0 and the total mean values of PSS for the cue condition are shown in Fig. 2 as a function of CLT. All values of PSS were positive and the PSSs decreased with CLT. The one-way repeated measures analysis of variance showed that the main effect of CLT was significant $(F(3,7)=17.3, p<0.01)$. The values of PSS in CLT of 150-200ms were significantly longer that those in CLT of 800 msec (Tukey's HSD test, $p<0.05$ ). The maximum PSS was about $50.6 \mathrm{msec}$ in CLT of $150 \mathrm{msec}$ and the minimum PSS was $4.15 \mathrm{msec}$ in CLT of $800 \mathrm{msec}$.

The results indicate that when the two targets were presented at the same time, participants perceived that the target presented in the same ear as a cue tone was perceived earlier than the target presented in the opposite ear. This means that the exogenous attention aroused by the preceding cue tone facilitated transmission of the target stimulus on the same side as the cue tone. However, it has been known that the prior entry effects found by TOJ task adopted in Experiment 1 might have been confounded by response bias (Pashler, 1998; Shore, Spence, \& Klein, 2001 ; 
Spence, Shore, \& Klein, 2001). Therefore, the possibility of contamination by response bias should be examined. In order to test this, in the next experiment, we asked participants to judge which target appeared to be presented later. In this case, if he truly perceived the target presented in the same ear as a cue tone earlier than that in the opposite ear when both targets were presented simultaneously, the same results as those of Experiment 1 will be obtained. If the facilitation effect by the cue tone was based on response bias, the results will be the opposite (Shore, Spence, \& Klein, 2001).

\section{Experiment 2}

Method

Participants

Three postgraduates, who did not participate in Experiment 1, participated in this experiment. All had normal hearing ability.

Procedure

Participants must judge which is the second target. They were instructed to push right key if they heard the right target later, and press the left key if they heard the left target later. The other things were equal to Experiment 1.

Results and Discussion

The same analysis as that of Experiment 1 was performed. The mean PSS across participants for the no-cue condition was 1.739 msec. For the cue condition, the mean PSSs as a function of CLT are 
plotted in Fig.3. The form of this figure is the same as Fig.2. The results are similar to those of Experiment 1. However, the maximum PSS was $80.2 \mathrm{msec}$ in CLT of $200 \mathrm{msec}$ and the minimum was $22.3 \mathrm{msec}$ in CLT of $800 \mathrm{msec}$. The facilitation effects were larger that those in Experiment 1. The one-way repeated measures analysis of variance showed that the main effect of CLT was significant $(F(3,7)=14.908, p<0.05)$. The values of PSS in CLT of 150-200ms were also significantly longer that those in CLT of $800 \mathrm{msec}$ (Tukey's HSD test, $\mathrm{p}<0.05$ ).

Since the results were almost the same as those of Experiment 1, we can think that the results of Experiment 1 were not contaminated by response. This indicates that the cue tone evoked participants' exogenous auditory attention, and that the spatial attention facilitated the transmission of the target tone at the same side, and/or inhibited the transmission of the target at the opposite side. The effects of the exogenous attention could be estimated to last for $400 \mathrm{msec}$ to $800 \mathrm{msec}$ after it was aroused by the cue tone. This duration of the effects was longer than those reported by Spence and Driver (1994), McDonald and Ward (1999). However, all results were consistent in the point that the effect of the exogenous attention diminished rapidly.

The results demonstrated that exogenous spatial attention aroused by a preceding cue tone could affect the speed of information transmission in the auditory system. This leads to the next question of whether spatial attention is more effective than spectral attention. It has been known that responses to tones of expected frequency are faster and more accurate than those of unexpected frequency (Greenberg \& Larkin, 1968; Scharf et al. 1987; Mondor \& Bregman, 1994). Thus, this question will be worth examined by an experiment. Therefore, in the next experiment, not only the cue positions but also the frequencies of tones were varied. In addition, we executed the condition that the cue tone was presented in center location, in order to investigate whether 
the frequency-consistent cue tone facilitates the processing of target tone.

\section{Experiment 3}

Method

Participants

Three undergraduates and postgraduates, who participated in Experiment 1, participated in this experiment. All had normal hearing ability.

Apparatus and materials

These were similar to those used in Experiment 1 and 2, except that cue tones and target tones were either $250 \mathrm{~Hz}$ or $1000 \mathrm{~Hz}$ pure tones.

Experimental conditions

In the no-cue condition, two target tones of different frequencies were presented dichotically. In half of the trials, a $250 \mathrm{~Hz}$ tone was presented to the right ear, and a $1000 \mathrm{~Hz}$ tone was presented to the left ear. In the remaining trials, presentation of tones was reversed. We call this variable right-target-first (RTF) condition. This was described with the frequency of the right side (e.g., the former case was described as RTF-250Hz and the latter case was described as RTF-1000Hz). SOAs between target tones were manipulated at eight levels in the same way as Experiment 1. Therefore, 16 kinds of stimuli were prepared for the no-cue condition. In the cue condition, a cue tone was presented with 
target tones. There were three kinds of variables with cue tones: cue position (CP), cue leading time (CLT) and cue frequency (CF) . The CLTs were the same as those in Experiment 1, whereas CPs were set at three levels; right, left and center. The CF is the frequency of the cue tone that was either 250 or $1000 \mathrm{~Hz}$. As a repeated measures design was adopted, participants received 384 trial conditions, composed of SOA ( 8 levels), RTF (2 levels), CP ( 3 levels), CF (2 levels), and CLT (4 levels).

Procedure

Each trial was carried out using the same methodology as Experiment 1. The experiment was composed of twelve sessions. The first session was a practice session for the no-cue condition, where 80 trials were performed with feedback of accuracy. In the following experimental sessions, feedback was not given. The second session was the experimental session for the no-cue condition, where 160 trials (10 trials $x 8$ SOAs $x 2$ RTFs) were performed. The following ten sessions were the experimental sessions for the cue condition, where 384 trials (8 SOAs $x 2$ RTFs $\mathrm{x} 2$ CFs $\mathrm{x} 3$ CPs $\mathrm{x} 4$ CLTs) were performed for each session. All other methodologies were the same as in Experiment 1.

Results and Discussion

The PSSs for each condition were estimated by the same method as in Experiment 1 and 2. The mean PSSs for the no-cue condition were $15.8 \mathrm{msec}$ in RTF-250Hz, and $-1.1 \mathrm{msec}$ in RTF-1000Hz conditions.

In the condition of center $\mathrm{CP}$, we can examine purely the cue frequency effect. In this condition, when the cue frequency is the same as that of right target, the PSSs were multiplied by -1 . The total mean values of PSS in center CP are shown in Fig $4 \mathrm{~A}$ as a function of CLT. In this figure, the positive value of PSS 
indicates that the frequency of first target was different from cue frequency and the frequency of second target was the same as that, and the negative PSS indicates the opposite order. The results shows that the PSSs in both CFs of $250 \mathrm{~Hz}$ and $1000 \mathrm{~Hz}$ were a little higher than 0 msec. This indicates that if the frequency of target was the same as cue frequency, irrespective of $250 \mathrm{~Hz}$ and $1000 \mathrm{~Hz}$, the listener perceived the target earlier than the opposite target, when the two targets were presented at the same time. However, this frequency effect was not significant statistically. An analysis of variance was performed on the PSSs with respect to $\mathrm{CF}$ and $\mathrm{CLT}$. There were no significant main effects and no significant interaction.

In the conditions of left and right CP, the PSSs for right CP were multiplied by -1 and both values of PSS were averaged. In the analysis, the condition of cue-target frequency relation (CTF) was adopted instead of RTF. The CTF indicates whether the frequency of target tone in the location of cue tone is the same as the frequency of cue tone (CTF: Same) or not (CTF: Different). The mean PSS in these conditions are shown in Fig $4 \mathrm{~B}$ as a function of CLT. The form of this figure is the same as Fig 2 and Fig. 3 . The parameter indicates the combination of CTF and CF. The result shows that most of PSSs were positive and that the uncued target tone should be presented earlier than the cued target tone to perceive both target tones as simultaneous, irrespective of CTF.

Next, an analysis of variance was performed on the PSSs with respect to CTF, CF and CLT. The main effects of CLT and CTF were significant (respectively, $F(3,16)=47.54, p<0.01 ; \quad F(1$, 16) $=6.36, \mathrm{p}<0.05)$. Subsequent tests showed that the PSSs in CLT of $150 \mathrm{msec}$ were significantly longer than those in CLT of $200 \mathrm{msec}$ (Tukey's HSD test, $\mathrm{p}<0.05$ ) and the PSSs in CLT of $150-200 \mathrm{msec}$ were significantly longer than those in CLT of $800 \mathrm{msec}$ (Tukey's HSD test, $\mathrm{p}<0.05)$. The effect of interaction between CLT and CTF was also significant $(F(3,16)=9.91, p<0.01)$. 
These analyses indicate the following results. (1) The cue frequency in the center had a little effect on PSS. (2) The target tone presented at the same side as the cue tone was perceived faster than the target at the opposite side. (3) The effects were maximized when the CLT was $150 \mathrm{msec}$, and they decreased as the CLT became longer. (4) This facilitation effect was slightly stronger when the frequencies of cue and target in the same location were identical. (5) The PSSs were shifted to a greater extent in the conditions where the cue location was consistent to the targets than in the conditions where the cue frequency was consistent to the targets. The major finding in this experiment was the result of (5), which indicates that attention toward a spatial location was more dominant than attention toward a certain frequency with respect to transmitting speed.

The results of Experiments 1 to 3 showed that the exogenous attention oriented to a certain direction affects the speed of following information transmission. The next question that we should consider concerns the stage in the auditory systems at which the effects occur. As described above, many previous researchers reported that spatial attention had no effects in the auditory detection tasks (Posner, 1978; Scharf et al. 1987; Buchtel \& Butter, 1988; Spence \& Driver, 1994). Rhodes (1987) argued that this was because the simple detection tasks were performed through non-spatial representation at early stages of the auditory system. McDonald and Ward (1999) argued that the tasks' spatial relevance induced to use location sensitive neurons in the later stages of the auditory system and these neurons are susceptible to the effects of spatial attention. Hence, we examined this point by using a different method in the following experiment.

It has been known that two tones of the same frequency are perceived as a fused tone if they are presented binaurally at the same time or with a slight asynchrony, but the impression of the fusion decreases if the asynchrony becomes longer. The perception 
of tone fusion depends on modality specific system, whereas receptor independent mechanisms are engaged in the perception of temporal order (Hirsh \& Sherrick, 1961; Pöppel, 1985; Pöppel, 1997). Provided the facilitation and inhibition in the transmitting speed occurred before the integration of binaural tones, it will be predicted that the perception of the fusion will be influenced by the presentation of a cue tone. That is, if two tones were presented simultaneously to both ears, and if one of the tones was given a benefit or a cost in transmitting speed by aroused attention in the initial stages, listeners will perceive them as two segregated tones. In contrast, if the tone were facilitated and inhibited at the later stages, listeners will perceive the tones as fused. In the following experiment, we conducted this tone fusion judgment task to investigate the stages at which the exogenous spatial attention was affected.

\section{Experiment 4}

Method

Participants

Five undergraduates and postgraduates participated in Experiment 4. One of them had participated in Experiment 1 and 3 . All of them had normal hearing ability.

Apparatus and materials

These were identical to those used in previous experiments, except that the sampling frequency of pure tones was changed to $48 \mathrm{kHz}$.

Experimental conditions 
Tone fusion judgment task was performed under the no-cue condition and the cue condition. In the no-cue condition, two target tones of the same frequency were presented to both ears. The target frequency was either $250 \mathrm{~Hz}$ or $1000 \mathrm{~Hz}(\mathrm{TF})$. The two target tones were presented with 13 variables of SOAs: $-12,-10,-8,-6,-4$, $-2,0,2,4,6,8,10,12 \mathrm{msec}$. Consequently, 26 kinds of stimuli were prepared for the No-cue condition. In the cue condition, a cue tone was presented before the target tones. The frequency of the cue tone (CF) was either 250 or $1000 \mathrm{~Hz}$ and the variables of cue positions (CPs) were right, left and center. TF and SOA variables were identical to the No-cue condition. Therefore, 156 different stimuli were used for the Cue condition.

\section{Procedure}

The experiment was performed using a similar methodology to previous experiments. Participants were required to judge whether the target tones were fused into a single tone or were separate, by pressing one of two keys. In the cue condition, a cue tone preceded target tones with $150 \mathrm{msec}$ asynchrony (CLT), and participants were instructed to ignore the cue tone. The experiment was composed of two sessions for the no-cue condition and 10 sessions for the cue condition. Each participant carried out 260 trials in each session of the no-cue condition, and 312 trials in each session of the cue condition. Therefore, each participant performed 20 trials under the same condition.

Results and Discussion

The mean probability of the response that participants perceived the targets as one fused tone was calculated under each SOA condition. The results for TFs of 250 and $1000 \mathrm{~Hz}$ are shown in 
Fig. 5A and Fig. 5B respectively. When the SOA was not zero msec in both figures, probabilities in conditions that cue frequency was the same as target frequency (250L, C, R condition in Fig. $5 \mathrm{~A}$, and 1000L, C, R condition in Fig. 5B) were lower than those in other conditions. In these conditions, participant's judgments were precise and cue frequency may have influence on tone fusion judgment. Nevertheless, probabilities were highest when the SOA was zero msec in all cue conditions, and probabilities decreased sharply with the increases of the absolute value of SOA. The fact that the peaks never shifted indicates that the participants perceived the targets as one fused tone when the targets were presented simultaneously, regardless of the cue tone. The results of this experiment showed that the preceding cue tones do not affect the auditory information processing at the initial stages. In other words, the results indicate that the effects observed in Experiments 1 to 3 occurred in the later stages after binaural information were integrated. These results support the hypothesis proposed by Rhodes (1987), McDonald and Ward (1999).

\section{General discussion}

The purpose of the present study was to examine the effects of exogenous spatial attention on the speed of information processing in the auditory system. By using the TOJ task in Experiment 1 to 3, we found that the exogenous attention aroused by the preceding cue tone facilitated transmission of the target stimulus on the same side as the cue tone. The effect was estimated to last for 400-800 msec, which almost corresponds to the results found by the measurement of discrimination reaction time (Spence \& Driver, 1994; McDonald \& Ward, 1999). Experiment 3 showed that the spatial-consistent cue was more effective than the frequency-consistent cue. This result suggested that with respect 
to the speed of information transmission, attention toward a spatial location was dominant when compared to attention to a certain frequency region. In addition, it was supposed from the results of Experiment 4 that the effect occurred in the stages after integration of binaural information.

Mondor, Zatorre, \& Terrio (1998) argued that: (1) selection of auditory information is performed by an attentional template that normally incorporates both location and frequency information, but (2) location information is not as dominant for auditory selective attention as for visual selective attention. The results of Experiment 3 in the present study appeared to be inconsistent with these arguments. From the present study, we could hypothesize that the location information is analyzed and this characteristic influences auditory transmitting speed. The evidence that endorses our hypothesis has been obtained already in the third experiment of Mondor et al. (1998). They showed that the spatially close distracter delayed the reaction to a greater degree than the spectrally similar distracter. These results indicate that the listeners selected the targets mainly based upon their spatial characteristic.

It has been generally considered that auditory attention toward a certain frequency region improves the sensitivity of stimulus detection in that region (e.g., Scharf et al. 1987), and that such an improvement is caused by the filtering mechanism at the initial stages of the auditory system (Scharf, Magnan, Collet, Ulmer \& Chays, 1994; Scharf, Magnan \& Chays, 1997). In contrast, it has been assumed that sensitivity to detect a stimulus at a certain location could not be improved by spatially oriented attention in audition (Scharf, 1988). Comparing previous findings with the results of the present experiments, we can assume that spatial attention is functionally different from spectral attention; the former plays a role in facilitation of the speed of information transmission, while the latter improves the 
sensitivity of stimulus detection. In addition, the stages where these two kinds of effects occur might be different; spectral attention at initial stages and spatial attention at later stages.

The present study adopted the TOJ task to circumvent the problem of interactive effects between response biases and attention. However, some researchers have pointed out that the prior entry effects found by this task have been confounded by response bias (Pashler, 1998; Shore, Spence, \& Klein, 2001 ; Spence, Shore, \& Klein, 2001). In order to test this, we compared two kinds of participant judgments: participants judged which target is presented first (Experiment 1) and second(Experiment 2). The results showed that they made almost the same judgments. This indicates that the experimental results in the present study were not contaminated by the response biases. However, these results do not perfectly rule out the role of response biases, because the left (or right) cues might have increased the probability of right (or left) first responses based on response biases in Experiment 2. The orthogonal method might be desirable to test the possibility. Still more, it remains a possibility that the results can be explained by the other non-attentional factor. The shift in PSS may have been due to non-attentional binding or sensory integration between the cue and target tones in the same location. This may have been because we used similar tones as cue and target stimuli in the experiments and the shifts were larger when the cue frequency was the same as the target frequency in Experiment 3. Nevertheless, this possibility will be ruled out because if such a binding or integration occurred, we could have obtained a similar effect in Experiment 4.

The shifts of PSSs in Experiment 1 to 3 indicate facilitation of the auditory information processing by exogenous spatial attention. However, there is still a question whether the exogenous spatial attention actually facilitated the information processing and actually sped up the transmission of auditory 
information. The same results might be obtained if information arising from the attended stimulus were amplified at the initial stages of processing without influencing the speed of transmission. However, this possibility would also be ruled out for the same reason as that mentioned above, i.e. that if such a process occurred, we could have obtained a similar effect in Experiment 4 .

In studies of the auditory system, it has been pointed out that the superior colliculus plays a role in exogenous orienting (Rhodes, 1987; Spence \& Driver, 1994; McDonald \& Ward, 1999). However, the stages where spatial attention has such effects are also still open to question. We do not know in detail at what stage of the auditory system exogenously shifted attention influences the judgment of TOJ tasks. The present study only revealed that it is not the stage before binaural tones are integrated. Nevertheless, the present research showed that the TOJ task and the fusion judgment task are promising tools in the study of auditory attention. Further investigations are needed to answer this and other questions about auditory spatial attention.

Acknowledgement We thank Dr. John McDonald for his helpful comments on earlier versions of this manuscript. 


\section{References}

Bregman, A. S. (1990). Auditory scene analysis: The perceptual organization of sound.

Cambridge, MA: MIT Press.

Buchtel, H. A., \& Butter, C. M. 1988 spatial attentional shifts: Implications for the role

of polysensory mechanism. Neuropsychologia, 26, 499-509.

Gibson, B.S., \& Egeth,H. (1994). Inhibition and disinhibition of return:

Evidence from temporal order judgments. Perception \& Psychophysics,

$56,669-680$.

Greenberg, G.Z., \& Larkin, W.D. (1968) Frequency-response characteristic of

auditory observers detecting signals of a single frequency in noise: The

probe-signal method. The Journal of the Acousitical Society of America, 44,

$1513-1523$.

Hirsh, I.J., \& Sherrick,C.E. (1961). Perceived order in different sense modalities.

Journal of Experimental Psychology, 62, 423-432.

Jaskowski, P. (1993) Selective attention and temporal-order judgment. Perception, 22, $681-689$.

McDonald, J.J., \& Ward,L.M. (1999) Spatial relevance determines facilitatory

and inhibition effects of auditory covert spatial orienting. Journal of

Experimental Psychology: Human Perception and Performance, 
$25, \quad 1234-$

1252 .

Mondor, T.A., \& Bregman,A. (1994) Allocating attention to frequency regions.

Perception \&Psychophysics, 56, 268-276.

Mondor, T.A., Zatorre, R. J., \& Terrio, N. A. (1998) Constraints on the selection of

auditory information. Journal of Experimental Psychology:

Human

Perception and Performance, 24, 66-79.

Pashler, H.E. (1998) The Psychology of Attention. Cambridge, MA: MIT Press.

Pöppel, E. (1985) Grenzen des Bewußtseins; Über Wirklichkeit und Welterfahrung.

stuttgart: DVA.

Pöppel, E. (1997) A hierarchical model of temporal perception.

Trends in Cognitive

Sciences, 1, 56-61.

Posner, M., I. (1978) Chronometric Explorations of Mind. Hillsdale, NJ : Erlbaum.

Quinlan, P. T., \& Bailey, P. J. (1995) An examination of attentional control in the auditory modality: Further evidence for auditory orienting . Perception and

Psychophysics, 57, 614-628.

Rhodes, G. (1987) Auditory attention and the representation of spatial information.

Perception and Psychophysics, 42, 1-14.

Sach, A.J., Hill, N.I., \& Baily, P.J. (2000) Auditory spatial attention using interaural

time differences. Journal of Experimental Psychology:

Human Perception

and Performance, 26, 717-729. 
Scharf, B. (1988) The role of listening in the measurement of hearing. In S. D. G.

Stephens (Ed.), Advances in Audiology (pp. 13-26). Basel: Karger.

Scharf, B. (1998) Auditory attention: The psychoacoustical approach. In $\mathrm{H}$. Pashler

(Ed.), Attention (pp. 75-117). Hove, UK: Psychology Press Ltd.

Scharf, B., Magnan, J., \& Chays, A. (1997) On the role of the olivocochlear bundle in

hearing: 16 case studies. Hearing Research, 103, 101-122. Scharf, B., Magnan, J., Collet, L., Ulmer, E., \& Chays, A. (1994) On the role of the

olivocochlear bundle in hearing: A case study. Hearing Research, 75, 11-16.

Scharf, B., Quigley, S., Aoki, C., Peachey, N., \& Reeves, A. (1987) Focused auditory

attention and frequency selectivity. Perception and Psychophysics, 42, 215-

223.

Shore,D.I., Spence, C., \& Klein, R.M. (2001) Visual prior entry. Psychological

Science, 12, 205-212.

Spence, C. J., \& Driver, J. (1994) Covert spatial orienting in audition: Exogenous and

endogenous mechanisms. Journal of Experimental

Psychology: Human

Perception and Performance, 20, 555-574.

Spence, C., Shore, D.I., \& Klein, R.M. (2001) Multisensory prior entry. Journal of

Experimental Psychology: General, 130, 799-832.

Sternberg, S., \& Knoll, R. L. (1973) The perception of temporal order: Fundamental 
issues and a general model. In S. Kornblum (Eds.), Attention and

Performance IV (pp.629-685). New York: Academic Press. Stelmach, L. B., \& Herdman, C. M. (1991) Directed Attention and Perception of

Temporal Order. Journal of Experimental Psychology: Human Perception

and Performance, 17, 539-550.

Titchener, E.B. (1908) Lectures on the Elementary Psychology of Feeling and

Attention. New York: Macmillan.

Treisman, A., \& Gelade, G. (1980) A feature-integration theory of attention. Cognitive

Psychology, 12, 97-136. 


\section{Figure Captions}

Fig. 1. Schematic representation of stimulus sequence used in Experiment $1 \& 2$.

Fig. 2. Mean PSS (msec) as a function of CLT(msec) in Experiment 1 . The positive PSS indicates that the uncued target is presented before the cued target. Error bars indicate \pm standard deviations.

Fig. 3. Mean PSS (msec) as a function of CLT (msec) in Experiment 2. The positive PSS indicates that the uncued target is presented before the cued target. Error bars indicate \pm standard deviations.

Fig. 4. Mean PSS (msec) as a function of CLT (msec) in Experiment 2. Panel A: The results for the condition of center CP. The parameter indicates the CF condition. The plus sign of PSS indicates that the frequency of first target was not the same as cue frequency (Different->Same). Panel B: The results for the conditions of left and right CPs. The parameter indicates the combination of CTF (Same, Different) and CF (250Hz and 1000Hz). The positive PSS indicates that the uncued target is presented before the cued target, and the negative PSS indicates the opposite order.

Fig. 5. Mean probability of 'one fused tone' response as a function of Stimuli Onset Asyncrony between two targets for each TF condition (panel A: TF-250Hz, panel B:TF-1000 Hz) in Experiment 3. The parameter indicates the combination of CP (Left, Right and Center) and CF (250Hz and $1000 \mathrm{~Hz})$. 


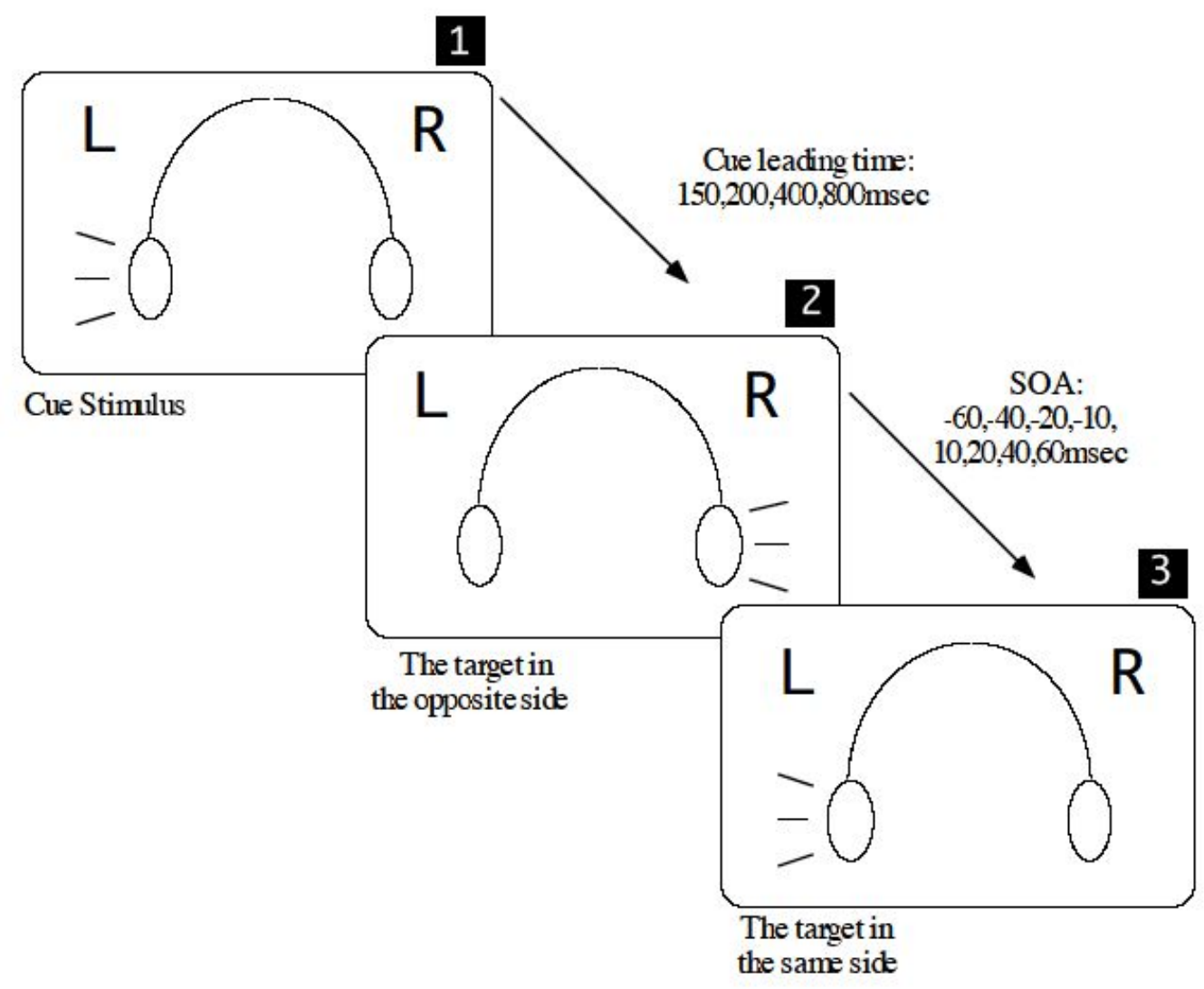

Fig.1 


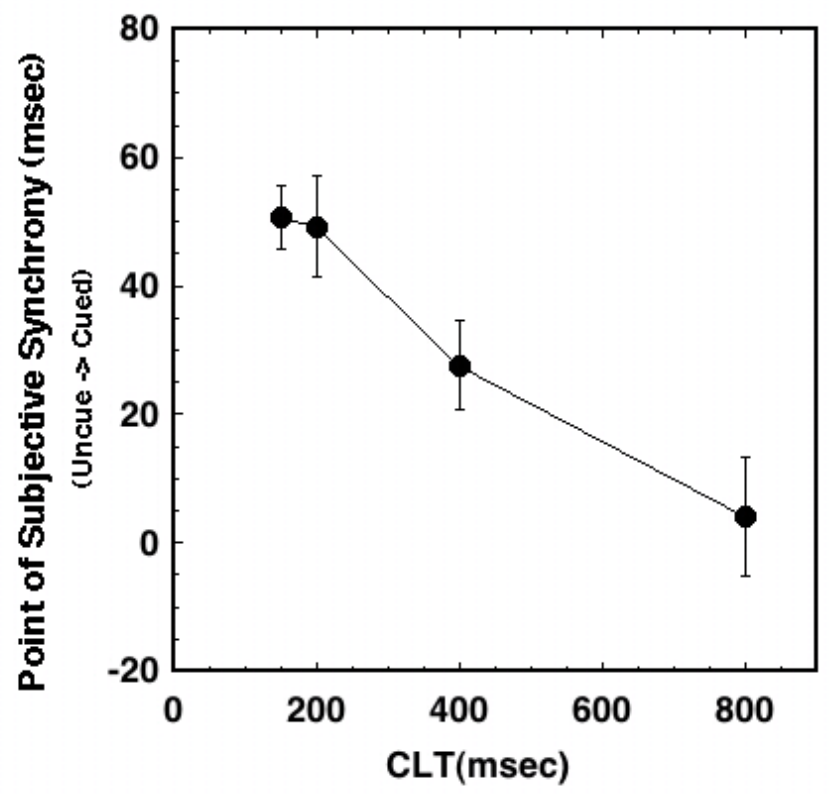

Fig.2 


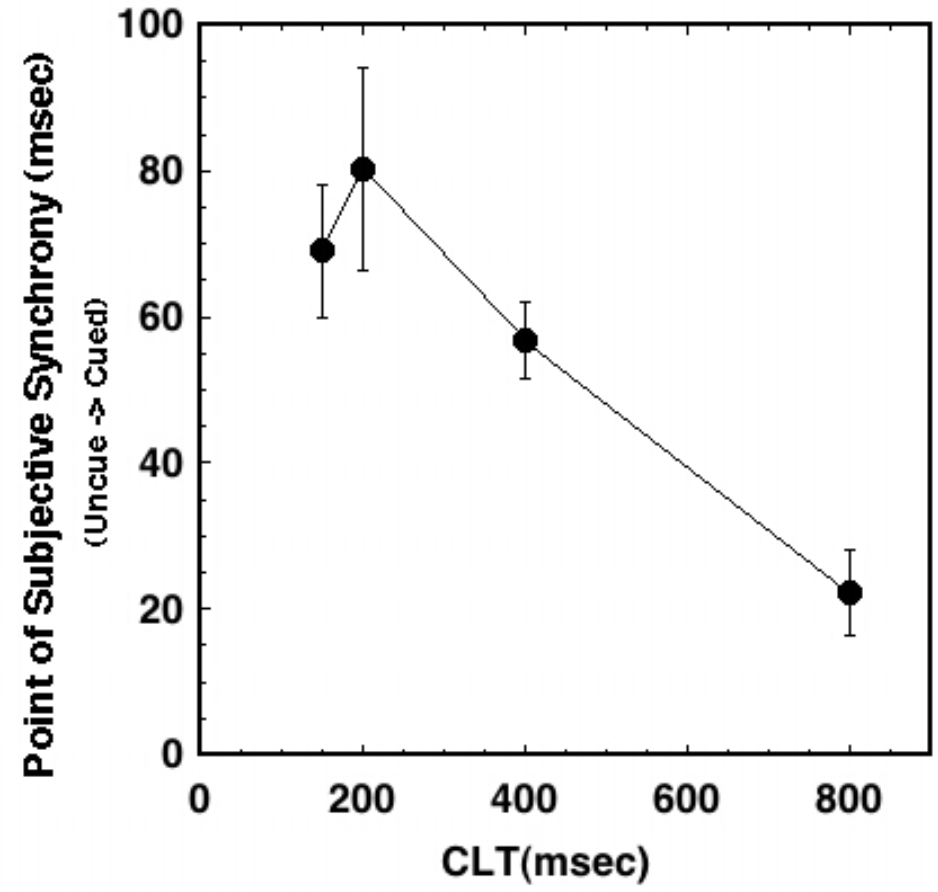

Fig.3 

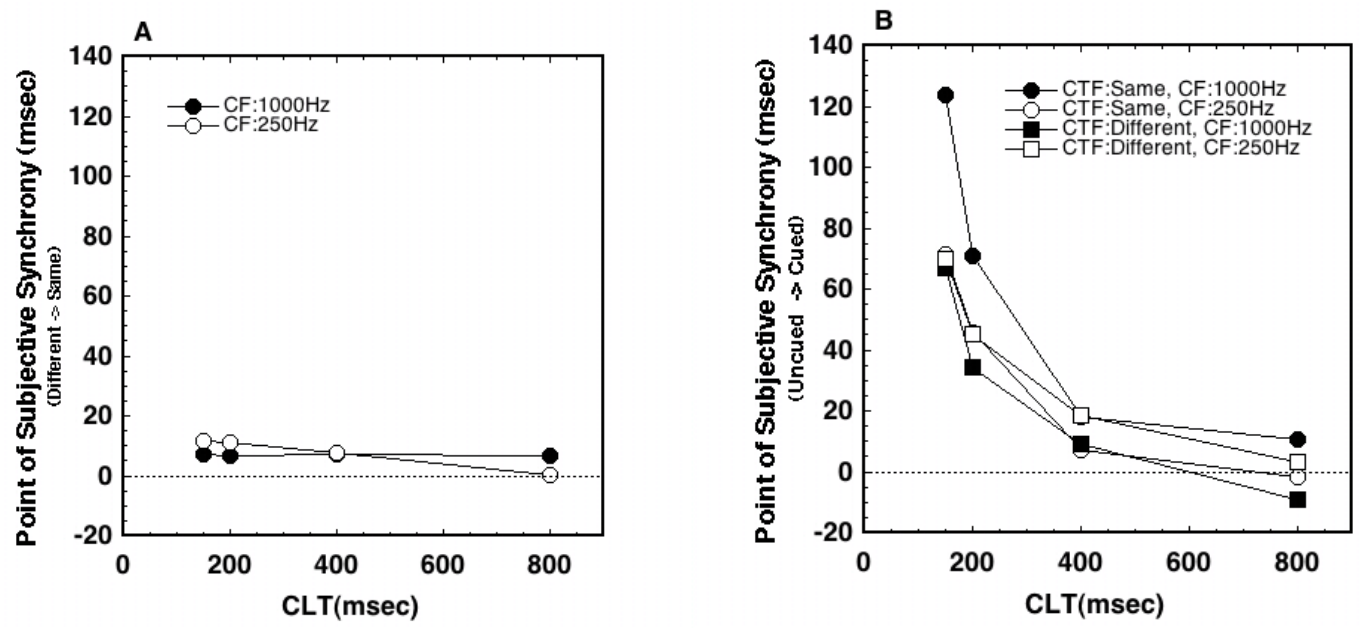

Fig4A\&Fig4B 

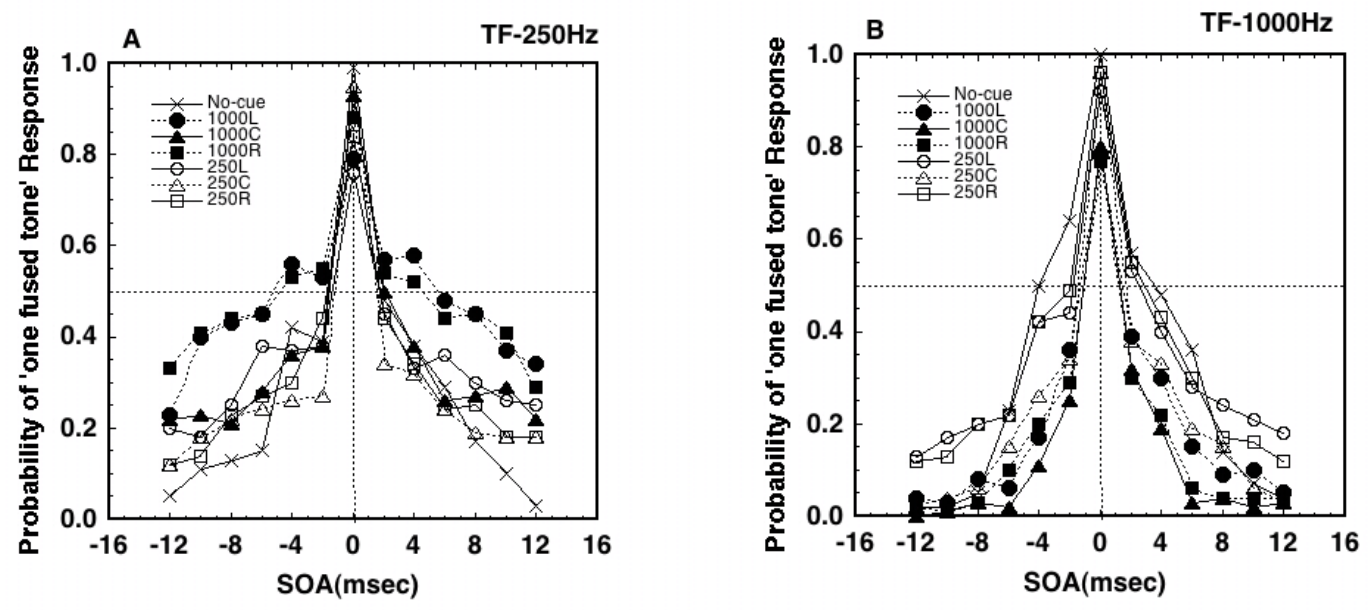

Fig5A\&Fig5B 